\title{
Angioedema Spotlight: A Closer Examination of Sacubitril/Valsartan Safety Results
}

\author{
Ryan E. Owens, PharmD, BCPS, and \\ Carrie S. Oliphant, PharmD, BCPS-AQ Cardiology
}

Incorporation of neprilysin inhibition into heart failure pharmacotherapy regimens has recently been recommended by U.S. guidelines, based on results from the PARADIGM-HF trial comparing sacubitril/ valsartan to enalapril. While most of the discussion has focused on efficacy, a closer examination of the safety results, particularly the incidence of angioedema during the run-in and double-blind periods, is also warranted. Although no major safety concerns were identified, an angioedema risk comparable to enalapril was found, primarily in the black population. Therefore, despite combination with an angiotensin receptor blocker, which historically has a lower incidence of angioedema, the addition of neprilysin inhibition yields an angioedema risk profile comparable to angiotensin converting enzyme (ACE) inhibitors. Clinicians should recognize this safety risk when prescribing sacubitril/valsartan and remain vigilant in counseling patients regarding the signs and symptoms of angioedema. As recommended by the guidelines, avoiding sacubitril/valsartan use concurrently or within 36 hours of the last dose of an ACE inhibitor or in patients with a history of angioedema is also crucial to minimize angioedema risk and prevent patient harm. (J Am Board Fam Med 2017;30:556-557.)

Keywords: Angioedema, Angiotensin Receptor Blockers, Heart Failure, Neprilysin

Heart failure management is embarking on a new era of pharmacotherapy with the discovery of neprilysin inhibition and subsequent approval of sacubitril/valsartan, a neutral endopeptidase inhibitor and an angiotensin receptor blocker (ARB), respectively. Inhibition of neprilysin, a neutral endopeptidase, increases levels of endogenous vasoactive peptides, such as bradykinin, which in turn can blunt the secondary effects of neurohormonal overactivation in heart failure. ${ }^{1}$ Given the reduced risk

This article was externally peer reviewed.

Submitted 2 March 2017; revised 10 April 2017; accepted 14 April 2017.

From the Department of Pharmacy, Clinical and Administrative Sciences, University of Oklahoma Health Sciences Center, Oklahoma City (REO); Clinical Pharmacy Specialist, Cardiology/Anticoagulation, Methodist University Hospital, Memphis, TN (CSO); and Department of Clinical Pharmacy, University of Tennessee College of Pharmacy, Nashville (CSO).

Funding: none.

Conflict of interest: none declared.

Corresponding author: Ryan E. Owens, PharmD, BCPS, Department of Pharmacy, Clinical and Administrative Sciences, University of Oklahoma Health Sciences Center, 1122 Northeast 13th St, Suite 4409, Oklahoma City, OK 73117 (E-mail: ryan-owens@ouhsc.edu). of death and heart failure-related hospitalizations in its landmark trial, Prospective Comparison of ARNI [Angiotensin Receptor Neprilysin Inhibitor] with ACEI [Angiotensin Converting Enzyme Inhibitor] to Determine Impact on Global Mortality and Morbidity in Heart Failure Trial (PARADIGM$\mathrm{HF})$, much of the spotlight has been placed on the efficacy results of sacubitril/valsartan. ${ }^{1}$ However, an emphasis on safety results, particularly risk of angioedema, should be of equal importance to clinicians, as vasoactive peptides have also been implicated in the occurrence of angioedema. ${ }^{2}$

Safety concerns with neprilysin inhibition first arose during early trials of omapatrilat, a combination angiotensin-converting enzyme (ACE) inhibitor and neprilysin inhibitor. A significant increase in angioedema was noted with omapatrilat compared with enalapril monotherapy, which ultimately led to termination of omapatrilat's development secondary to safety concerns. ${ }^{3}$ Investigators subsequently combined neprilysin inhibition with ARB therapy, given the decreased incidence of angioedema with ARBs compared with ACE inhibitors. ${ }^{4,5}$ While early phase II studies examining sacu- 
bitril/valsartan indicated a low risk of angioedema in approximately 650 patients, they were short (only 12 weeks). ${ }^{6,7}$ The phase III study, PARADIGM-HF, examined 8442 patients at a median follow-up of 27 months, which helped better estimate angioedema incidence, a secondary safety end point of the trial. ${ }^{1}$ Patients with a history of angioedema were excluded from the trial at enrollment.

Overall, $0.45 \%$ of the sacubitril/valsartan group and $0.24 \%$ of the enalapril group experienced angioedema during the treatment period $(P=.13) .{ }^{1}$ Approximately $50 \%$ of the cases were managed during the treatment period with no treatment or with the use of antihistamines only. Ultimately, permanent discontinuation due to angioedema was required in 7 patients taking sacubitril/valsartan and 4 patients taking enalapril, whereas a temporary interruption with a negative rechallenge was experienced in five and two patients respectively. ${ }^{8}$ It is also important to note that an additional 25 angioedema cases (10 patients taking sacubitril/valsartan and 15 patients taking enalapril) occurred during the run-in period, which led to drug discontinuation in 8 of the sacubitril/valsartan patients and all 15 enalapril patients. ${ }^{8}$

Results for the black population are of particular interest when examining angioedema subanalysis results. Overall, 5 of the 213 black patients (2.3\%) taking sacubitril/valsartan compared with 1 of the 214 black patients $(0.5 \%)$ taking enalapril experienced angioedema; 1 of the patients in the sacubitril/valsartan group required subsequent hospitalization. ${ }^{8}$ Assessing specifically the US black population in PARADIGM-HF, 3 of the 54 patients $(5.6 \%)$ treated with sacubitril/valsartan experienced angioedema, compared with none in the enalapril group. ${ }^{8}$ Regarding severity, 1 of the 3 patients required hospitalization but experienced no airway compromise. All the North American patients who experienced angioedema with sacubitril/valsartan during the study period were black. ${ }^{8}$

Overall, sacubitril/valsartan therapy exhibits a risk of angioedema similar to that observed with ACE inhibitors. Clinicians should not be misled by its combination with valsartan, given that ARBs historically display a low angioedema incidence, as the addition of neprilysin inhibition raises the angioedema risk to a level similar to that of other ACE inhibitors. ${ }^{5}$ Heart failure guideline safety recommendations, such as avoiding administration of an Angiotensin Receptor Neprilysin Inhibitor (ARNI) concomitantly or within 36 hours of the last dose of an ACE inhibitor and avoiding its use in patients with a history of angioedema, should be followed closely to prevent potential patient harm. ${ }^{9}$ Clinicians should remain vigilant in monitoring and counseling patients on the signs and symptoms of angioedema throughout sacubitril/ valsartan therapy, especially black patients, as they seem to be at highest risk and may have been underrepresented as a whole in PARADIGM-HF.

To see this article online, please go to: http://jabfm.org/content/ 30/4/556. full.

\section{References}

1. McMurray JJ, Packer M, Desai AS, et al. Angiotensin-neprilysin inhibition versus enalapril in heart failure. N Engl J Med 2014;371:993-1004.

2. Fryer RM, Segreti J, Banfor PN. Effect of bradykinin metabolism inhibitors on evoked hypotension in rats: rank efficacy of enzymes associated with bradykinin-mediated angioedema. Br J Pharmacol 2008; 153:947-55.

3. Kostis JB, Packer M, Black HR, Schmieder R, Henry $\mathrm{D}$, Levy E. Omapatrilat and enalapril in patients with hypertension: the omapatrilat cardiovascular treatment vs. enalapril (OCTAVE) trial. Am J Hypertens 2004; 17:103-11.

4. Gu J, Noe A, Chandra P, et al. Pharmacokinetics and pharmacodynamics of LCZ696, a novel dual-acting angiotensin receptor-neprilysin inhibitor (ARNi). J Clin Pharmacol 2010;50:401-14.

5. Toh S, Reichman ME, Houstoun M, et al. Comparative risk for angioedema associated with the use of drugs that target the renin-angiotensin-aldosterone system. Arch Intern Med 2012;172:1582-9.

6. Solomon SD, Zile M, Pieske B, et al. The angiotensin receptor neprilysin inhibitor LCZ696 in heart failure with preserved ejection fraction: a phase 2 double-blind randomised controlled trial. Lancet 2012;380:1387-95.

7. Senni M, McMurray JJ, Wachter R, et al. Initiating sacubitril/valsartan (LCZ696) in heart failure: results of TITRATION, a double-blind, randomized comparison of two uptitration regimens. Eur J Heart Fail 2016;18:1193-202.

8. Center for Drug Evaluation and Research. Application number 207620Orig1s000. Medical review(s): LCZ696 (Entresto). Available from: http:// www.accessdata.fda.gov/drugsatfda_docs/nda/2015/ 207620 Orig1s000MedR.pdf. Accessed 29 Aug 2016.

9. Yancy CW, Jessup M, Bozkurt B, et al. 2016 ACC/ AHA/HFSA focused update on new pharmacological therapy for heart failure: an update of the 2013 ACCF/ AHA guideline for the management of heart failure: a report of the American College of Cardiology/American Heart Association Task Force on Clinical Practice Guidelines and the Heart Failure Society of America. J Am Coll Cardiol 2016;68:1476-88. 